\title{
Compartimentos da Matéria Orgânica de Solos sob Floresta no Estado do Acre
}

\author{
Ademir Fontana ${ }^{1}$, Marcos Gervasio Pereira ${ }^{2}$, Thiago Andrade Bernini ${ }^{3}$, \\ Lúcia Helena Cunha dos Anjos², Paulo Guilherme Salvador Wadt ${ }^{4}$, \\ Lauana Lopes dos Santos ${ }^{5}$ \\ ${ }^{1}$ Pesquisa, Embrapa Solos, Rio de Janeiro/RJ, Brasil \\ ${ }^{2}$ Departamento de Solos, Universidade Federal Rural do Rio de Janeiro - UFRRJ, Seropédica/RJ, Brasil \\ ${ }^{3}$ Instituto Federal de Educação, Ciência e Tecnologia do Rio de Janeiro - IFRJ, Pinheiral/RJ, Brasil \\ ${ }^{4}$ Pesquisa, Embrapa Rondônia, Porto Velho/RO, Brasil \\ ${ }^{5}$ Universidade Federal dos Vales do Jequitinhonha e Mucuri - UFVJM, Diamantina/MG, Brasil
}

\begin{abstract}
RESUMO
O objetivo deste trabalho foi quantificar os diferentes compartimentos da matéria orgânica de solos sob floresta no estado do Acre. Perfis de solo foram descritos e coletados nos terços superior, terço médio e terço inferior da paisagem localizados nos municípios de Sena Madureira, Manoel Urbano e Feijó, no estado do Acre. Os solos foram caracterizados quanto à composição química, física, frações húmicas e frações oxidáveis. Os teores de carbono orgânico estão concentrados nos horizontes superficiais dos solos, destacados pelo decréscimo abrupto em relação aos demais horizontes. Os teores da argila e de cátions atuam de forma mais efetiva na dinâmica da matéria orgânica em detrimento à posição na paisagem e classe de solo. As análises multivariadas indicam maior similaridade entre os pontos de Sena Madureira (T1) e de Feijó (T3), exceto o ponto do terço superior de Feijó, que é semelhante ao Manoel Urbano (T2).
\end{abstract}

Palavras-chave: atributos dos solos, frações orgânicas, horizonte superficial.

\section{Organic Matter Compartments from under Forest Soils in the Acre State}

\begin{abstract}
This study aimed to quantify the different compartments of organic matter from under forest soils in Acre state. Soil profiles were described and sampled on shoulder, backslope and footslope landscape positions located in the counties of Sena Madureira, Manoel Urbano and Feijó, Acre state. Soils were characterized by chemically and physically attribute, humic and oxidizable fractions. The organic carbon is concentrated in the superficial soil horizons, highlighted by the sharp decrease in relation to other horizons. The clay and cations act more effectively on organic matter dynamics than the landscape position and soil class. Multivariate analyzes indicate high similarity between the sites of Sena Madureira (T1) and Feijó (T3), except the shoulder site from Feijó, which is similar to Manoel Urbano (T2).
\end{abstract}

Keywords: soil attributes, organic fractions, surface horizon. 


\section{INTRODUÇÃO}

$\mathrm{Na}$ bacia do Acre, que abrange grande parte do território acreano, região sudoeste da Amazônia, é comum a ocorrência de solos com teores de cálcio e magnésio trocáveis muito variáveis (grande amplitude), por vezes coincidente com altos teores de alumínio, e comumente associados àa presença de minerais de argila de atividade alta e caráter expansivo (Gama et al., 1992; Wadt, 2002; Araújo et al., 2004).

Em relação à matéria orgânica do solo (MOS), com base nos teores de carbono orgânico ( $\mathrm{C}$ org), tem-se observado teores significativos e concentrados nos horizontes superficiais, estes com espessura bastante reduzida e, ainda, com o decréscimo abrupto para os horizontes subsuperficiais. Este padrão foi observado pelo trabalho realizado no alto Purus, no estado do Acre, por Volkoff et al. (1989).

Desta forma, presume-se que os fatores que controlam a dinâmica da MOS da região estão relacionados à presença de cátions trocáveis e à mineralogia da fração argila. Para Kononova (1966) e Duchaufour (1977), a presença de teores altos de cátions trocáveis e mineralogia 2:1 favorece a formação de complexos organominerais de elevada estabilidade na superfície do solo.

O entendimento da dinâmica da MOS, com seus compartimentos e suas correlações podem servir como indicadores de mudanças ambientais que levem à manutenção, aumento ou perda, resultando na melhor compreensão da interação entre usos/coberturas e solos nos ecossistemas do Bioma Amazônia. Nesse sentido, as avaliações mais específicas do componente orgânico, como a análise dos diferentes compartimentos, representados pelas frações húmicas e as frações oxidáveis, podem fornecer informações além daquelas apresentadas pela análise dos teores de $\mathrm{C}$ org.

Diante desse pressuposto, o objetivo desse trabalho foi quantificar os diferentes compartimentos da matéria orgânica de solos sob floresta no estado do Acre.

\section{MATERIAL E MÉTODOS}

Foram selecionadas áreas localizadas nos municípios de Sena Madureira (S 903.237 e W 68 57.796), Manoel Urbano (S 843.740 e W 69 31.242) e Feijó (S 831.985 e W 6959.184 ), no estado do Acre, região sudoeste amazônica.
A geologia da região está relacionada à Formação Solimões e sedimentos Cenozóicos (Cavalcante, 2006). A unidade geomorfológica é a Depressão do Juruá-Iaco, sendo o relevo composto por topos convexos e aguçados, com declividade média a forte e com dissecação convexa e aguçada, com altitudes de 150 a 440 m (Acre, 2006). A vegetação dominante é a de Floresta Aberta com Bambu, associada à Floresta Aberta com Palmeiras.

O clima pela classificação de Köppen é do tipo Aw, definido como tropical com estação seca no inverno, e Am, tropical chuvoso (Brasil, 1976). Os índices pluviométricos anuais são superiores a $1.600 \mathrm{~mm}$, com duas estações definidas: o período chuvoso de novembro a abril, e outro menos chuvoso, de junho a setembro. A temperatura média anual é em torno de $24,5^{\circ} \mathrm{C}$, com máxima de $32{ }^{\circ} \mathrm{C}$ (Acre, 2006).

Em cada área foi estabelecida uma topossequência sob vegetação nativa de floresta e foram abertas trincheiras em três pontos da encosta: terço superior, terço médio e terço inferior. A distância entre cada ponto amostrado dentro e entre as topossequências é variada e influenciada por variações pontuais do relevo. A coleta e descrição dos perfis foi realizada segundo Santos et al. (2005), e a classificação dos solos conforme Santos et al. (2013).

As amostras dos solos foram avaliadas quanto ao carbono orgânico (Yeomans \& Bremner, 1988), $\mathrm{pH}$ (água), complexo sortivo ( $\mathrm{Ca}, \mathrm{Mg}, \mathrm{Na}, \mathrm{K}$ ), acidez potencial $(\mathrm{H}+\mathrm{Al})$ e análise granulométrica (Donagemma et al., 2011).

As frações húmicas foram quantificadas com base na solubilidade da matéria orgânica, conforme método proposto por Benites et al. (2003). Foram separadas três frações: ácidos fúlvicos, obtida com solução de $\mathrm{NaOH}$ 0,1 mol L ${ }^{-1}$; ácidos húmicos, obtida com solução de $\mathrm{NaOH} 0,1 \mathrm{~mol} \mathrm{~L}^{-1}$ e precipitado em meio ácido diluído; e a humina, insolúvel em solução de $\mathrm{NaOH}$ $0,1 \mathrm{~mol} \mathrm{~L}^{-1}$ e obtida como resíduo.

As frações oxidáveis foram obtidas segundo o método proposto por Chan et al. (2001), obtendo-se quatro frações: $\mathrm{F}$ : carbono oxidado em $3 \mathrm{~mol} \mathrm{~L}^{-1} \mathrm{de}$ $\mathrm{H}_{2} \mathrm{SO}_{4} ; \mathrm{F} 2$ : diferença do carbono oxidado em 6 e $3 \mathrm{~mol} \mathrm{~L}^{-1}$ de $\mathrm{H}_{2} \mathrm{SO}_{4}$; F3: diferença do carbono oxidado em 9 e $6 \mathrm{~mol} \mathrm{~L}^{-1}$ de $\mathrm{H}_{2} \mathrm{SO}_{4}$; F4: diferença do carbono oxidado em 12 e $9 \mathrm{~mol} \mathrm{~L}^{-1}$ de $\mathrm{H}_{2} \mathrm{SO}_{4}$.

Os dados obtidos dos horizontes A foram submetidos a análise de correlação simples, multivariadas de 
componentes principais (com rotação varimax) e de agrupamentos hierárquicos. Os dados para as análises multivariadas foram padronizados (média zero e variância um) devido à variação da amplitude pelas diferentes unidades de medida. Todas as análises foram realizadas pelo programa Statistica 7 (Statsoft Inc, 2004).

\section{RESULTADOS E DISCUSSÃO}

Os dados morfológicos dos perfis destacam nas topossequências os horizontes $\mathrm{A}$ com pequenas espessuras, com no máximo $10 \mathrm{~cm}$, onde somados com os horizontes $\mathrm{AB}$ chegam a $26 \mathrm{~cm}$ (Tabela 1). Os horizontes subsuperficiais apresentam ampla variação de espessura e está relacionada ao grau de desenvolvimento dos solos, com horizontes Bi, Btf e Bg mais espessos, com exceção o perfil T1-S, que apresenta horizonte $\mathrm{C}$ a $5 \mathrm{~cm}$ de profundidade.

De forma geral, os teores de argila tendem a aumentar em profundidade, exceto para o perfil T1-I (Tabela 1). Os valores de $\mathrm{pH}$ estão entre 3,9 e 6,7, sendo os maiores valores nos horizontes $\mathrm{A}$ e $\mathrm{AB}$, que são reflexo da presença de cátions básicos no complexo sortivo. Nesses horizontes são observados os maiores valores

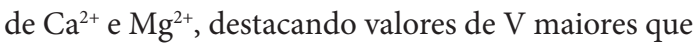
50\% (eutrófico). A exceção, os perfis T2-S, T3-S e T3-M (distrófico) (Tabela 1). Os horizontes $\mathrm{A}$ e $\mathrm{AB}$ apresentam baixos teores de $\mathrm{Al}^{3+}$, sendo entre 0,0 e 1,0 $\mathrm{cmol}_{\mathrm{c}} \mathrm{kg}^{-1}$. Nos horizontes subsuperficiais B e C, observa-se grande variação, com teores entre 0,0 e 14,5 $\mathrm{cmol}_{\mathrm{c}} \mathrm{kg}^{-1} \mathrm{e}$, na maioria, superiores a 4,0 $\mathrm{cmol}_{\mathrm{c}} \mathrm{kg}^{-1}$ (Tabela 1 ).

Os teores de $\mathrm{C}$ org nos horizontes $\mathrm{A}$ variam de 26,5 a 78,4 $\mathrm{g} \mathrm{kg}^{-1}$, enquanto nos horizontes $\mathrm{AB}$ de 3,2 a 11,2 $\mathrm{g} \mathrm{kg}^{-1}$, e nos horizontes B e C de 1,3 a 6,7 $\mathrm{g} \mathrm{kg}^{-1}$ (Tabela 1). Em relação aos teores de carbono orgânico das frações húmicas, o C-HUM foi predominante, com valores entre 50 e $73 \%$ do C org do solo, podendo ser avaliado também pelos baixos valores da relação C-EA/C-HUM (Tabela 2). Ainda, os solos apresentam valores baixos do C-FAF e do C-FAH, sendo exceção a T1-M e T3-I. De maneira geral, o C-FAH é maior em detrimento ao C-FAF e assim indicados pelos valores superiores a 1,0 da relação C-FAH/C-FAF (Tabela 2).

Para solos com teores de $\mathrm{C}$ org concentrados nos horizontes superficiais e com baixos valores da relação C-EA/C-HUM, destaca-se o alto potencial de acúmulo de carbono proporcionado pela elevada estabilidade da matéria orgânica com a matriz mineral do solo (Fontana et al., 2010a).

Volkoff et al. (1989) verificaram padrão semelhante em Argissolos Amarelos e Vermelho-Amarelos do alto Purus, estado do Acre, com maiores teores nos horizontes $\mathrm{A}$, com decréscimo abrupto e redução em profundidade, além da pequena espessura dos horizontes superficiais. Entretanto, em uma floresta tropical densa (floresta perenifólia) desenvolvida sob um Argissolo Amarelo distrófico, no município de Sena Madureira, Araújo et al. (2004) observaram na profundidade de 0-10 cm teores de $\mathrm{C}$ org entre 2,6 e $15,7 \mathrm{~g} \mathrm{~kg}^{-1}$, e na profundidade de $10-60 \mathrm{~cm}$ de 4,3 a $5,9 \mathrm{~g} \mathrm{~kg}^{-1}$.

Em condições climáticas diferentes, na região semiárida do Rio Grande do Norte, porém com características semelhantes dos solos, com altos teores de $\mathrm{Ca}^{2+}$ e argila do tipo 2:1, Volkoff \& Cerri (1980) observaram que o $\mathrm{C}$ org se concentrava na superfície do solo, associado também ao predomínio da humina. Padrão semelhante foi observado por Miranda \& Ferreira (1999) em perfis de solo da zona da mata norte do estado de Pernambuco. Corrêa et al. (2003) também destacam o predomínio da fração humina em solo da região das várzeas no município de Souza, estado da Paraíba, assim como Cunha \& Ribeiro (1998) em perfis de solo da região de Irecê, no estado da Bahia.

Segundo Kononova (1966) e Duchaufour (1977), a estabilidade da matéria orgânica em solos é atribuída à presença de cátions divalentes ou trivalentes, os quais fazem a ponte entre a matéria orgânica e a matriz mineral com minerais de argila do tipo 2:1, formando complexos organominerais de elevada estabilidade na superfície do solo. Como possível relação, destaca-se a correlação positiva e significativa entre o $\mathrm{Ca}^{2+}$ e C org e C-HUM (Figura 1).

Quanto à relação C-FAH/C-FAF, a observação de valores superiores a 1,0 indica alta taxa de humificação da MOS, pois, segundo Moreno (1996), baixos valores estão relacionados a uma baixa taxa de humificação, sendo observados em locais com aporte constante de material orgânico. Para solos sob Mata Atlântica, comparativamente, os valores da relação C-FAH/C-FAF apresentam padrão inverso, com valores inferiores a 0,8 nas camadas superficiais (Fontana et al., 2010b, 2011).

Pela distribuição das frações oxidáveis, destaca-se o predomínio da F1 (27 a 79\%), seguido da F3 > F2 > F4 (Tabela 3). Às frações F1 e F2 
Tabela 1. Classes e atributos dos solos dos municípios de Sena Madureira (T1), Manoel Urbano (T2) e Feijó (T3). Table 1. Soils classes and attributes from Sena Madureira (T1), Manoel Urbano (T2) and Feijó (T3) counties.

\begin{tabular}{|c|c|c|c|c|c|c|c|c|c|c|c|}
\hline \multirow{2}{*}{ Posição } & \multirow{2}{*}{$\begin{array}{c}\text { Classes de } \\
\text { Solo }\end{array}$} & \multirow{2}{*}{ Horizonte } & Prof. & pH & $\mathrm{Ca}^{2+}$ & $\mathrm{Mg}^{2+}$ & $\mathrm{Al}^{3+}$ & $\mathbf{V}$ & $\mathbf{m}$ & Corg & Argila \\
\hline & & & $\mathrm{cm}$ & água & \multicolumn{3}{|c|}{$-\mathrm{cmol}_{\mathrm{cg}} \mathrm{kg}^{-1}-$} & \multicolumn{2}{|c|}{$-\%-$} & \multicolumn{2}{|c|}{$\mathrm{g} \mathrm{kg}^{-1}$} \\
\hline \multirow{3}{*}{$\mathrm{T} 1-\mathrm{S}$} & \multirow{3}{*}{$\begin{array}{l}\text { Vertissolo } \\
\text { Háplico } \\
\text { Órtico típico }\end{array}$} & A & $0-5$ & 6,0 & 11,0 & 12,0 & 0,0 & 87 & 0 & 36,7 & 240 \\
\hline & & CA & $5-14$ & 3,9 & 4,9 & 11,6 & 5,4 & 59 & 25 & 6,7 & 336 \\
\hline & & $\mathrm{CV}_{1}$ & $14-34$ & 4,2 & 8,2 & 15,3 & 9,4 & 61 & 29 & 2,9 & 549 \\
\hline \multirow{4}{*}{$\mathrm{T} 1-\mathrm{M}$} & \multirow{4}{*}{$\begin{array}{l}\text { Cambissolo } \\
\text { Háplico Ta } \\
\text { Eutrófico } \\
\text { típico }\end{array}$} & A & $0-3$ & 5,1 & 8,0 & 8,5 & 0,1 & 71 & 1 & 29,2 & 293 \\
\hline & & $\mathrm{AB}$ & $3-12$ & 4,5 & 5,3 & 8,2 & 0,6 & 69 & 4 & 9,7 & 335 \\
\hline & & BA & $12-21$ & 4,2 & 4,5 & 5,5 & 2,6 & 55 & 21 & 6,6 & 426 \\
\hline & & $\mathrm{Bi}_{1}$ & $21-42$ & 4,2 & 6,4 & 5,8 & 3,1 & 62 & 20 & 4,8 & 435 \\
\hline \multirow{4}{*}{ T1-I } & \multirow{4}{*}{$\begin{array}{l}\text { Cambissolo } \\
\text { Háplico Ta } \\
\text { Eutrófico } \\
\text { típico }\end{array}$} & A & $0-8$ & 6,0 & 16,2 & 15,7 & 0,0 & 87 & 0 & 46,0 & 203 \\
\hline & & $\mathrm{AB}$ & $8-18$ & 5,4 & 10,6 & 12,5 & 0,1 & 84 & 0 & 11,2 & 386 \\
\hline & & BA & $18-43$ & 4,6 & 8,9 & 14,2 & 4,5 & 73 & 16 & 3,2 & 458 \\
\hline & & $\mathrm{Bi}_{1}$ & $43-58$ & 4,3 & 3,5 & 15,0 & 8,4 & 58 & 31 & 2,7 & 363 \\
\hline \multirow{5}{*}{$\mathrm{T} 2-\mathrm{S}$} & \multirow{5}{*}{$\begin{array}{l}\text { Argissolo } \\
\text { Vermelho- } \\
\text { Amarelo } \\
\text { Alítico } \\
\text { plintossólico }\end{array}$} & A & $0-3$ & 5,9 & 11 & 2,6 & 0,0 & 65 & 0 & 59,9 & 189 \\
\hline & & $\mathrm{AB}$ & $3-9$ & 4,7 & 4,0 & 0,8 & 0,5 & 49 & 9 & 7,1 & 226 \\
\hline & & $\mathrm{BA}$ & $9-20$ & 4,2 & 2,3 & 2,0 & 3,0 & 36 & 41 & 4,3 & 323 \\
\hline & & $\mathrm{Bt}$ & $20-56$ & 3,9 & 1,2 & 7,6 & 14,0 & 30 & 61 & 4,6 & 697 \\
\hline & & $\mathrm{Btf}_{1}$ & $56-83$ & 4,0 & 1,2 & 6,6 & 14,5 & 27 & 65 & 3,6 & 614 \\
\hline \multirow{4}{*}{ T2-M } & \multirow{4}{*}{$\begin{array}{l}\text { Cambissolo } \\
\text { Háplico Ta } \\
\text { Eutrófico } \\
\text { típico }\end{array}$} & A & $0-6$ & 5,9 & 14,4 & 3,8 & 0,0 & 74 & 0 & 59,1 & 320 \\
\hline & & $\mathrm{AB}$ & $6-18$ & 5,0 & 8,5 & 2,5 & 0,7 & 68 & 6 & 5,9 & 308 \\
\hline & & BA & $18-29$ & 4,1 & 4,7 & 8,9 & 8,7 & 51 & 39 & 4,1 & 506 \\
\hline & & $\mathrm{Bi}_{1}$ & $29-43$ & 4,1 & 4,5 & 9,5 & 12,1 & 45 & 46 & 3,2 & 552 \\
\hline \multirow{4}{*}{ T2-I } & \multirow{4}{*}{$\begin{array}{l}\text { Gleissolo } \\
\text { Háplico Ta } \\
\text { Eutrófico } \\
\text { típico }\end{array}$} & A & $0-6$ & 6,7 & 23,0 & 5,0 & 0,0 & 88 & 0 & 78,4 & 278 \\
\hline & & $A B$ & $6-21$ & 6,6 & 17,5 & 5,3 & 0,0 & 92 & 0 & 5,1 & 351 \\
\hline & & BA & $21-42$ & 5,7 & 18,2 & 8,8 & 0,6 & 87 & 2 & 3,0 & 537 \\
\hline & & $\mathrm{Bg}$ & $42-81$ & 5,5 & 12,6 & 7,4 & 2,2 & 80 & 10 & 1,3 & 425 \\
\hline \multirow{6}{*}{ T3-S } & \multirow{6}{*}{$\begin{array}{l}\text { Argissolo } \\
\text { Vermelho } \\
\text { Alítico } \\
\text { plintossólico }\end{array}$} & A & $0-3$ & 5,8 & 16,1 & 1,4 & 0,0 & 75 & 0 & 77,8 & 187 \\
\hline & & $\mathrm{AB}_{1}$ & $3-15$ & 5,0 & 2,2 & 0,8 & 0,2 & 51 & 5 & 4,7 & 232 \\
\hline & & $\mathrm{AB}_{2}$ & $15-26$ & 4,5 & 1,0 & 1,5 & 1,0 & 36 & 28 & 3,5 & 239 \\
\hline & & $\mathrm{BA}$ & $26-40$ & 4,4 & 1,0 & 1,2 & 5,5 & 26 & 71 & 2,3 & 387 \\
\hline & & $2 \mathrm{Bt}$ & $40-88$ & 4,7 & 1,1 & 2,0 & 10,8 & 18 & 78 & 3,0 & 671 \\
\hline & & $2 \mathrm{Btf}$ & $88-148$ & 4,8 & 0,8 & 1,6 & 13,1 & 13 & 84 & 1,4 & 548 \\
\hline \multirow{6}{*}{ T3-M } & \multirow{6}{*}{$\begin{array}{l}\text { Argissolo } \\
\text { Acinzentado } \\
\text { Distrófico } \\
\text { plintossólico }\end{array}$} & A & $0-5$ & 5,0 & 8,5 & 0,2 & 0,1 & 65 & 1 & 26,5 & 248 \\
\hline & & $A B$ & $5-11$ & 5,0 & 4,4 & 0,6 & 0,2 & 68 & 4 & 3,2 & 224 \\
\hline & & BA & $11-21$ & 5,0 & 5,0 & 1,1 & 0,7 & 68 & 10 & 3,1 & 282 \\
\hline & & $\mathrm{Bt}_{1}$ & $21-29$ & 4,9 & 4,9 & 3,1 & 2,4 & 60 & 23 & 3,4 & 348 \\
\hline & & $\mathrm{Bt}_{2}$ & $29-41$ & 4,9 & 2,5 & 8,8 & 9,9 & 42 & 47 & 2,6 & 597 \\
\hline & & $\mathrm{Btf}_{1}$ & $41-54$ & 4,9 & 1,5 & 8,7 & 13,2 & 34 & 56 & 2,1 & 611 \\
\hline & Cambissolo & A & $0-10$ & 4,9 & 12,8 & 2,7 & 0,1 & 71 & 1 & 29,6 & 404 \\
\hline T3-I & Háplico Ta & $\mathrm{AB}$ & $10-18$ & 5,1 & 9,9 & 3,2 & 0,8 & 74 & 6 & 6,7 & 383 \\
\hline $13-1$ & Eutrófico & BA & $18-35$ & 4,9 & 11,0 & 4,7 & 4,3 & 66 & 21 & 3,8 & 454 \\
\hline & típico & $\mathrm{Bi}$ & $35-66$ & 5,3 & 16,3 & 12,3 & 9,6 & 65 & 25 & 2,0 & 506 \\
\hline
\end{tabular}

$\mathrm{T}$ = topossequência; Prof. = profundidade; $\mathrm{S}=$ terço superior; $\mathrm{M}=$ terço médio; $\mathrm{I}=$ terço inferior; $\mathrm{V}=$ saturação por bases; $\mathrm{m}$ = saturação por alumínio; $\mathrm{C}$ org = carbono orgânico.

atribui-se a presença de compostos orgânicos de maior labilidade, estando relacionada com a fração leve livre da matéria orgânica e ao aporte constante dos resíduos vegetais da floresta, enquanto as frações F3 e F4 estão relacionadas aos compostos de maior estabilidade da matéria orgânica (Chan et al., 2001; Maia et al., 2007; Rangel et al., 2008).

Pela análise de correlação, observa-se que as quatro frações oxidáveis apresentam correlação positiva com o C-HUM $(0,67$ a $0,82 p<0,05)$. A correlação 
Tabela 2. Teores de carbono orgânico e frações húmicas do horizonte A dos solos dos municípios de Sena Madureira (T1), Manoel Urbano (T2) e Feijó (T3).

Table 2. Organic carbon and humic fractions tenors of A soil horizon from Sena Madureira (T1), Manoel Urbano (T2) and Feijó (T3) counties.

\begin{tabular}{|c|c|c|c|c|c|c|c|c|c|}
\hline \multirow{2}{*}{ Posição } & Corg & C-FAF & C-FAH & C-HUM & \multirow{2}{*}{$\begin{array}{c}\text { C-FAH/ } \\
\text { C-FAF }\end{array}$} & \multirow{2}{*}{$\begin{array}{c}\text { C-EA/ } \\
\text { C-HUM }\end{array}$} & FAF & FAH & \multirow[t]{2}{*}{ HUM } \\
\hline & $\mathrm{g} \mathrm{kg}^{-1}$ & \multicolumn{3}{|c|}{$\mathrm{g} \mathrm{kg}^{-1}$} & & & \multicolumn{2}{|c|}{$\%$} & \\
\hline T1-S & 36,7 & 2,6 & 3,4 & 25,7 & 1,3 & 0,2 & 7 & 9 & 70 \\
\hline T1-M & 29,2 & 3,7 & 4,4 & 14,7 & 1,2 & 0,6 & 13 & 15 & 50 \\
\hline T1-I & 46,0 & 4,2 & 3,9 & 30,8 & 0,9 & 0,3 & 9 & 8 & 67 \\
\hline T2-S & 59,9 & 3,3 & 4,4 & 37,5 & 1,3 & 0,2 & 6 & 7 & 63 \\
\hline T2-M & 59,1 & 3,6 & 5,3 & 43,3 & 1,5 & 0,2 & 6 & 9 & 73 \\
\hline T2-I & 78,4 & 5,6 & 5,7 & 52,0 & 1,0 & 0,2 & 7 & 7 & 66 \\
\hline T3-S & 77,8 & 5,0 & 6,7 & 52,0 & 1,4 & 0,2 & 6 & 9 & 67 \\
\hline T3-M & 26,5 & 2,3 & 4,4 & 15,9 & 1,9 & 0,4 & 9 & 17 & 60 \\
\hline T3-I & 29,6 & 7,0 & 7,0 & 15,8 & 1,0 & 0,9 & 24 & 24 & 53 \\
\hline
\end{tabular}

$\mathrm{C}-\mathrm{FAF}=$ carbono das frações ácidos fúlvicos; $\mathrm{C}-\mathrm{FAH}=$ carbono das frações ácidos húmicos; C-HUM = carbono da fração humina; C-EA = extrato alcalino (C-FAF + C-FAH).

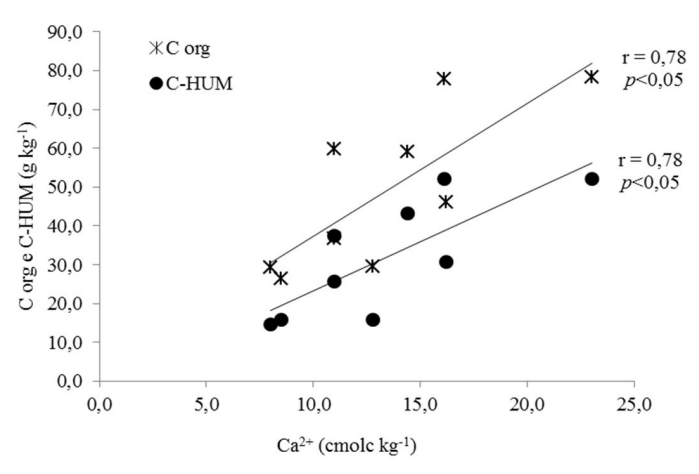

Figura 1. Dispersão do $\mathrm{Ca}^{2+}$ com C org e C-HUM dos horizontes $\mathrm{A}$ das três topossequências.

Figure 1. Distribution of $\mathrm{Ca}^{2+}$ with $\mathrm{C}$ org and C-HUM of the A horizons from three topossequences.

positiva da F1 e F2 com C-HUM nestes solos pode ser devido à precipitação da matéria orgânica fresca carboxilada pela ação dos cátions trocáveis, como $\mathrm{Ca}^{2+}$ e minerais de argila do tipo 2:1, que propiciam a formação de complexos organominerais, no entanto, de fraca estabilidade, o que pode observado pela dispersão com ultrassom e separação por densidade (Duchaufour, 1977). Este padrão está em acordo com o relatado no método de fracionamento proposto por Benites et al. (2003), que destaca que a fração humina pode conter compostos orgânicos de menor solubilidade em meio alcalino, como ceras e demais compostos hidrofóbicos, além da matéria orgânica leve estabilizada nos complexos organominerais.
A ação dos cátions e minerais de argila do tipo 2:1 favorece a polimerização de pequenas moléculas orgânicas solúveis, levando à insolubilização da MOS, e ainda pela formação de pontes com a matéria orgânica fresca pela demetoxilação e oxidação da lignina, gerando aumento dos grupos funcionais carboxílicos (Duchaufour, 1977).

Com a análise exploratória multivariada de componentes principais, os atributos apresentam relações/pesos distintos em cada componente principal (CP), assim como a relação entre atributos. Para o CP1, tem-se o $\mathrm{Ca}^{2+}, \mathrm{Al}^{3+}, \mathrm{pH}, \mathrm{C}$ org, C-HUM, F1, F2, F3 e F4 (Tabela 4). Enquanto para o CP2, o C-FAF, C-FAH, e no $\mathrm{CP} 3 \mathrm{o} \mathrm{Mg}^{2+}$ e $\mathrm{SB}$. A argila e C-EA/C-HUM tem relação com o CP1 e CP2 e C-FAH/C-FAF no CP2 e CP3.

Em uma segunda análise, é possível observar como cada atributo influencia os pontos amostrados (Figura 2). Pela análise da distribuição dos pontos de cada topossequência no CP1 e CP2, destacam-se o T2-I e T3-S relacionados aos altos teores de $\mathrm{Ca}^{2+} \mathrm{e}$ $\mathrm{C}$ org, e em sentido oposto ao T1-M e o T3-I, estes relacionados aos altos valores de C-EA/C-HUM, destacando ainda o T3-I com altos teores de argila, o que levou ao maior afastamento aos demais pontos (Figuras 2 e 3 ).

Outra observação, pelo cruzamento dos escores do CP1 e CP3, reforça o observado anteriormente, mas destaca ainda a relação do T3-M com o alto valor do C-FAH/C-FAF. O T1-S e T1-I se destacam dos demais 
Tabela 3. Frações oxidáveis do horizonte A dos solos dos municípios de Sena Madureira (T1), Manoel Urbano (T2) e Feijó (T3).

Table 3. Oxidizable fractions of A soils horizons from Sena Madureira (T1), Manoel Urbano (T2) and Feijó (T3) counties.

\begin{tabular}{|c|c|c|c|c|c|c|c|c|c|}
\hline \multirow{2}{*}{ Posição } & Corg & F1 & F2 & F3 & F4 & F1 & F2 & F3 & F4 \\
\hline & $\mathrm{g} \mathrm{kg}^{-1}$ & \multicolumn{4}{|c|}{$\mathrm{g} \mathrm{kg}^{-1}$} & \multicolumn{4}{|c|}{$\%$} \\
\hline T1-S & 36,7 & 28,5 & 1,5 & 4,5 & 1,31 & 79 & 4 & 12 & 4 \\
\hline T1-M & 29,2 & 11,3 & 3,0 & 3,0 & 1,18 & 48 & 12 & 12 & 25 \\
\hline T1-I & 46,0 & 16,5 & 12,8 & 11,3 & 0,93 & 39 & 30 & 26 & 3 \\
\hline $\mathrm{T} 2-\mathrm{S}$ & 59,9 & 19,5 & 10,5 & 19,5 & 1,33 & 38 & 20 & 38 & 2 \\
\hline T2-M & 59,1 & 25,5 & 6,0 & 13,5 & 1,47 & 43 & 10 & 23 & 23 \\
\hline T2-I & 78,4 & 27,0 & 19,5 & 15 & 1,01 & 36 & 26 & 20 & 16 \\
\hline T3-S & 77,8 & 20,3 & 15,0 & 30,0 & 1,35 & 27 & 20 & 40 & 12 \\
\hline T3-M & 26,5 & 8,25 & 7,5 & 6,0 & 1,89 & 35 & 32 & 25 & 6 \\
\hline T3-I & 29,6 & 8,25 & 6,0 & 7,5 & 1,00 & 33 & 24 & 30 & 12 \\
\hline
\end{tabular}

Tabela 4. Carregamento dos atributos dos solos e dos compartimentos da matéria orgânica das três topossequências nos componentes principais (CP).

Table 4. Loading of soils attributes and organic matter compartments of the three toposequences in the principal components (CP).

\begin{tabular}{|cccccccc|}
\hline Atributo & CP1 & CP2 & CP3 & Atributo & CP1 & CP2 & CP3 \\
\hline Argila & $-0,52$ & 0,64 & 0,02 & C-FAH & 0,18 & 0,90 & $-0,34$ \\
\hline $\mathrm{Ca}^{2+}$ & 0,73 & 0,48 & 0,39 & C-HUM & 0,99 & 0,15 & 0,00 \\
\hline $\mathrm{Mg}^{2+}$ & $-0,12$ & $-0,34$ & 0,90 & C-FAH/C-FAF & $-0,06$ & $-0,50$ & $-0,79$ \\
$\mathrm{Al}^{3+}$ & $-0,85$ & 0,25 & $-0,33$ & C-EA/C-HUM & $-0,81$ & 0,58 & $-0,08$ \\
\hline $\mathrm{SB}$ & 0,43 & 0,08 & 0,87 & $\mathrm{~F} 1$ & 0,74 & $-0,24$ & 0,33 \\
\hline $\mathrm{pH}$ & 0,85 & $-0,12$ & 0,46 & $\mathrm{~F} 2$ & 0,74 & 0,36 & 0,06 \\
\hline $\mathrm{C}$ org & 0,97 & 0,22 & $-0,02$ & $\mathrm{~F} 3$ & 0,81 & 0,20 & $-0,31$ \\
\hline C-FAF & 0,06 & 0,96 & 0,20 & $\mathrm{~F} 4$ & 0,58 & 0,42 & $-0,10$ \\
\hline
\end{tabular}
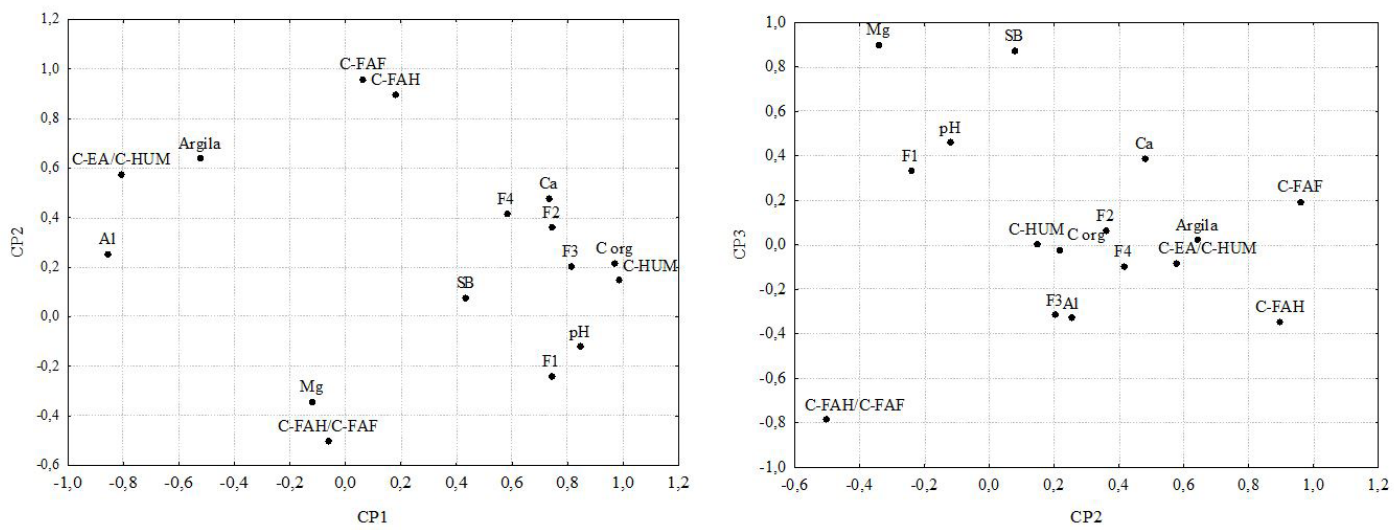

Figura 2. Distribuição dos atributos dos solos e dos compartimentos da matéria orgânica das três topossequências nos componentes principais.

Figure 2. Distribution of the soils attributes and organic matter compartments of the three toposequences in the principal components. 

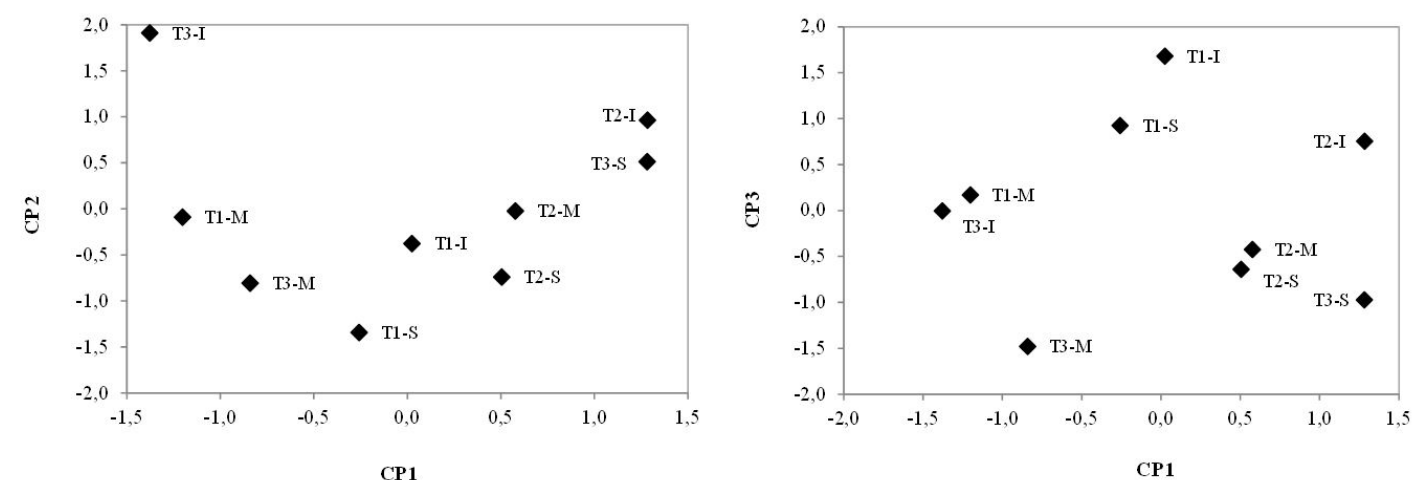

Figura 3. Distribuição dos pontos em cada topossequência nos componentes principais. Figure 3. Distribution of sites from each toposequence in the principal components.

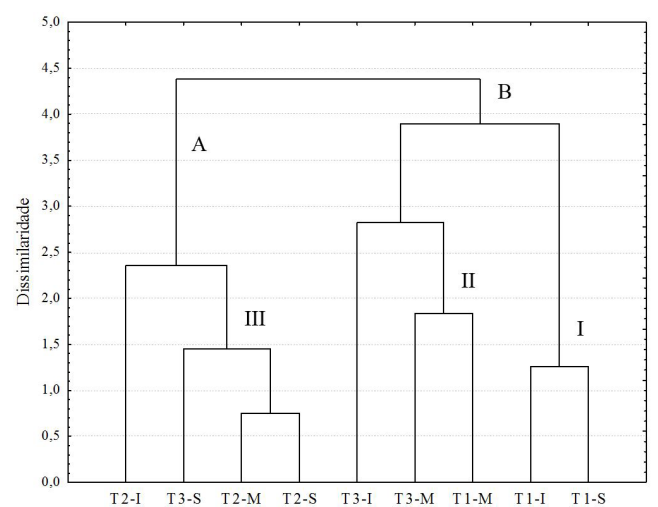

Figura 4. Agrupamento pela dissimilaridade dos pontos de cada topossequência pelo método de Ward's e distância Euclidiana. A e B - agrupamentos primários. I, II e III - agrupamentos secundários.

Figure 4. Grouping by dissimilarity of the sites from each toposequence by Ward's method and Euclidean distance. A and B - primary grouping. I, II and III - secondary grouping.

pelos altos teores de $\mathrm{Mg}^{2+}$ e $\mathrm{SB}$ e o T2-I com $\mathrm{pH}$ e $\mathrm{SB}$ (Figuras 2 e 3). Os pontos T2-S e T2-M ocupam posição intermediária, o que significa menor variância dos atributos estudados em relação aos demais pontos.

A tendência de distribuição dos pontos amostrados da análise dos componentes principais pode ser observada pela análise de agrupamento, conforme o dendograma de dissimilaridade (Figura 4). O gráfico destaca 2 grupos distintos (A e B), e ainda os grupos I, II e III com cada ponto amostrado. No grupo I estão os pontos T1-S e T1-I; grupo II o T1-M, T3-M, e ainda com certa semelhança o T3-I; grupo III em nível de maior similaridade o T2-S e T2-M, sendo estes com certa semelhança ao T3-S e seguindo com o T2-I.

\section{CONCLUSÕES}

Os teores de carbono orgânico estão concentrados nos horizontes superficiais dos solos, destacados pelo decréscimo abrupto em relação aos demais horizontes subsuperficiais.

Os teores da argila e de cátions atuam de forma mais efetiva na dinâmica da matéria orgânica em detrimento à posição na paisagem e classe de solo.

As análises multivariadas indicam maior similaridade entre os pontos de Sena Madureira (T1) e de Feijó (T3), exceto o ponto do terço superior de Feijó, que é semelhante ao Manoel Urbano (T2).

\section{STATUS DA SUBMISSÃO}

Recebido: 23 ago., 2013

Aceito: 12 jul., 2016

\section{AUTOR(ES) PARA CORRESPONDÊNCIA}

\section{Ademir Fontana}

Pesquisa, Embrapa Solos, Rua Jardim Botânico, 1024, Bairro Jardim Botânico, CEP 22460-000, Rio de Janeiro, RJ, Brasil

e-mail: ademir.fontana@embrapa.br 


\section{REFERENNCIAS}

Acre. Governo do Estado do Acre. Programa Estadual de Zoneamento Ecológico Econômico do Estado do Acre. Zoneamento Ecológico-Econômico - ZEE/AC 2 a fase [CDROM]. Rio Branco: SECTMA; 2006.

Araújo EA, Lani JL, Amaral EF, Guerra A. Uso da terra e propriedades físicas e químicas de Argissolo Amarelo distrófico na Amazônia Ocidental. Revista Brasileira de Ciência do Solo 2004; 28(2): 307-315. http://dx.doi. org/10.1590/S0100-06832004000200009.

Benites VM, Madari BE, Machado PLOA. Extração e fracionamento quantitativo de substâncias húmicas do solo: um procedimento simplificado de baixo custo. Rio de Janeiro: Embrapa Solos; 2003. Comunicado Técnico n. 16.

Brasil. Projeto Radambrasil. Rio Branco: geologia, geomorfologia, pedologia, vegetação, uso potencial da terra. Rio de Janeiro: Divisão de Publicação; 1976. Folhas SC - Levantamento de recursos naturais n. 19.

Cavalcante LM. Relatório sobre a Geologia do Estado do Acre. Rio Branco: SEMA: IMAC; 2006.

Chan KY, Bowman A, Oates A. Oxidizable organic carbon fractions and soil quality changes in an oxic paleustalf under different pasture ley. Soil Science 2001; 166(1): 61-67. http://dx.doi.org/10.1097/00010694-200101000-00009.

Corrêa MM, Ker JC, Mendonça ES, Ruiz HA, Bastos RS. Atributos físicos, químicos e mineralógicos de solos da região de várzeas de Souza (PB). Revista Brasileira de Ciência do Solo 2003; 27(2): 311-324. http://dx.doi. org/10.1590/S0100-06832003000200011.

Cunha TJF, Ribeiro LP. Qualidade e relações pedogenéticas da matéria orgânica de alguns solos da região do Irecê(BA). Revista Brasileira de Ciência do Solo 1998; 22(4): 693-704. http://dx.doi.org/10.1590/S0100-06831998000400015.

Donagemma GK, Campos DVB, Calderano SB, Teixeira WG, Viana JHM. Manual de métodos de análises de solos. 2. ed. Revista. Rio de Janeiro: Embrapa Solos; 2011. 230 p. Embrapa Solos Documentos n. 132.

Duchaufour P. Pedology. London: George Allen \& Unwin Publication; 1977.

Fontana A, Pereira MG, Anjos LHC, Benites VM. Quantificação e utilização das frações húmicas como característica diferencial em horizontes diagnósticos de solos brasileiros. Revista Brasileira de Ciência do Solo 2010a; 34(4): 1241-1257. http://dx.doi.org/10.1590/ S0100-06832010000400023.

Fontana A, Pereira MG, Brito RJ, Loss A, Benites VM. Caracterização de substâncias húmicas da camada superficial do solo sob diferentes coberturas vegetais. Magistra 2010b; 22(1): 49-56.

Fontana A, Silva CF, Pereira MG, Loss A, Brito RJ, Benites VM. Avaliação dos compartimentos da matéria orgânica em área de Mata Atlântica. Acta Scientiarum Agronomy 2011; 33(3): 545-550.

Gama JRNF, Kusaba T, Ota T, Amano Y. Influência de material vulcânico em alguns solos do Estado do Acre. Revista Brasileira de Ciência do Solo 1992; 16(1): 103-106.

Kononova MM. Soil organic matter: its nature, its role in soil formation and in soil fertility. 2. ed. Oxford: Pergamon Press; 1966.

Maia SMF, Xavier FAS, Senna OT, Mendonça ES, Araujo JA Fo. Organic carbono pools in a Luvisol under agroforestry and conventional farming systems in the semi-arid region of Ceará, Brazil. Agroforestry Systems 2007; 71(1): 127-138. http://dx.doi.org/10.1007/s10457-007-9063-8.

Miranda CSS, Ferreira MGVX. Caracterização de solos com A chernozêmico na zona da mata norte do estado de Pernambuco. Revista Brasileira de Ciência do Solo 1999; 23(1): 107-120. http://dx.doi.org/10.1590/S010006831999000100014 .

Moreno JL. La matéria orgânica em los agrossistemas. Madri: Ministéria Agricultura; 1996.

Rangel OJP, Silva CA, Guimarães PTG, Guilherme LRG. Frações oxidáveis do carbono orgânico de latossolo cultivado com cafeeiro em diferentes espaçamentos de plantio. Ciência e Agrotecnologia 2008; 32(2): 429-437. http://dx.doi.org/10.1590/S1413-70542008000200013.

Santos HG, Jacomine PKT, Anjos LHC, Oliveira VA, Lumbreras JF, Coelho MR et al. Sistema brasileiro de classificação de solos. 3. ed. Brasília: Embrapa; 2013. 353 p.

Santos RD, Lemos RC, Santos HG, Ker JC, Anjos LHC. Manual de descrição e coleta de solo no campo. 5. ed. Viçosa: Sociedade Brasileira de Ciência do Solo; Rio de Janeiro: Embrapa Solos; 2005.

Statsoft Inc. Statistica for Windows: computer program manual [online]. Tulsa: Statsoft Inc.; 2004. [citado em 2013 ago. 6]. Disponível em: http://www.statsoft.com.

Volkoff B, Cerri CC. Comparação de húmus de um solontchak, um rendzina e um solo litólico da região semiárida do Rio Grande do Norte. Revista Brasileira de Ciência do Solo 1980; 4(1): 49-56.

Volkoff B, Melfi AJ, Cerri CC. Solos Podzólicos e Cambissólicos Eutróficos do Alto Purus (Estado do Acre). Revista Brasileira de Ciência do Solo 1989; 13(3): 363-372.

Wadt PGS. Manejo de solos ácidos do Estado do Acre. Rio Branco: Embrapa Acre; 2002. Documentos n. 79.

Yeomans JC, Bremner JM. A rapid and precise method for routine determination of organic carbon in soil. Communications in Soil Science and Plant Analysis 1988; 19(13): 1467-1476. http://dx.doi.org/10.1080/00103628809368027. 